\title{
A Maintenance Strategy of Improving the Reliability of Relay Protection Based on Markov Model
}

\author{
Ruizhe Wang ${ }^{1}$ \\ ${ }^{1}$ School of Electrical and Electric Engineering, North China Electric Power University, Beijing, \\ 102206, China
}

Key words: Relay Protection, Reliability, Markov Model, Optimal Maintenance Cycle

\begin{abstract}
Power system, which has large-scale and complex structure,plays a significant role in national economy development.However, the system components will occur various malfunction or be an abnormal operation state due to various man-made and natural factors. This paper aims to ensure the power grid's safe operation by improving the reliability of relay protection which acts as a key component of the power system. We firstly construct the relay protection reliability evaluation index.Then we obtain the optimal maintenance cycle of protection devices based on Markov model to enhance furthermore to guarantee security safe and stable operation of power systems.
\end{abstract}

\section{Introduction}

Electric power industry is the foundation of national economy, and an important pillar industry.Relay protection is the first defense line to protect the safety of power system. Its fast and reliable action will effectively curb the deterioration of the system state to ensure the safety and reliability of power system operation.

At present, most of the power companies in our country still use the mode of regular maintenance, which means using same time interval in a fixed period of time for maintenance, such as the overhaul time scheduled for one year four times. However, fixed maintenance time will cause a lot of problems such as excessive maintenance and less maintenance. Excessive maintenance often results in the waste of human and material resources, and less maintenance makes the reliability of the equipment decreasing and the crisis of power system safe operation.

Based on the current situation of relay protection, the reliability of relay protection can be improved through mathematical modeling and big data processing and analysis to calculate the optimal maintenance cycle of relay protection.

\section{Commonly Reliability Indexes}

Reliability Degree and Fault Probability Density. [1] Under specified conditions and prescribed time, the probability of the equipment to complete its required function is defined as the devices reliability degree. Based on Probability Theory, if $X$ is a random variable to describe the equipment life, the distribution function of random variable $X$ is:

$$
F(t)=P\{X \leq t\}
$$

According to the definition of reliability and equipment life, the equipment reliability can be described as $R(t)$.

$$
R(t)=P\{X>t\}=1-F(t)=\bar{F}(t)
$$


So, the fault probability density function of the equipment is:

$$
f(t)=F^{\prime}(t)-R^{\prime}(t)(2-3)
$$

Fault Rate. Fault rate is the probability of fault in the next unit time on the condition that equipment in the ${ }^{[0, t]}$ period time does not occur to fault. It can be express as following:

$$
\lambda(t)=\lim _{\Delta t \rightarrow 0} \frac{P([t, t+\Delta t])}{\Delta t}
$$

In fact, the function $\lambda(t)$ describes the reliability variation law of operating equipment due to the existence of the fault probability density function $f(t)$.

Maintenance Degree and Repair Rate. Maintenance degree is the probability that the using product can maintain or restore to complete the required functional status in accordance with the provisions of the maintenance's procedures and methods. Maintenance degree is denoted asM(t). It describes the difficulty of equipment maintenance. Its expression is:

$$
M(t)=P(T \subset[0, t])=\int_{0}^{t} g(t) d t
$$

In the function (2-5), the $T$ is expressed as the maintenance time of fault equipment, $P(T \subset[0, t])$ is expressed as the probability of equipment repairing in ${ }^{[0, t]}$. The function $g(t)_{\text {is }}$ expressed as the probability density function of maintenance.

Repair rate is defined as the probability that product can be repaired completely in per unit time after the moment in which has not yet been repaired to express the fault equipment repairing process. Using the maintenance probability density function $g(t)$ describes the probability changing in the repair processes.

$$
\mu(t)=\lim _{\Delta t \rightarrow 0} \frac{P([t, t+\Delta t] \mid t)}{\Delta t}=\frac{M^{\prime}(t)}{1-M(t)}=\frac{g(t)}{1-M(t)}
$$

Availability Rate and Unavailability Rate. Availability rate is the normal running time in proportion of the total time[2].

$$
A=\frac{M T T F}{M T T F+M T T R}
$$

So, the unavailability rete is equal to $1-A$.

If the life and maintenance time distribution of the system components are exponential distribution, it can be described by a special stochastic class processes, the Markov process.

For the reliability analysis of the Markov type repairable system, the following features are mainly studied:

The reliability degree $R(t)$ at specified time in system and the unreliability degree $F(t)$

The average time until the first fault MTTF in the system。

The transient availability $A(t)$ and unavailability $Q(t)$ at the specified time in the system 
The steady-state value of availability $A(\infty)$ and unavailability in the system

The Mean time to failure of the system MTBF

\section{Markov Model}

The homogeneous Markov state space method is one of the most common methods in engineering reliability analysis based on constant failure rate. This method can be used by dividing the state of relay protection in different states, based on the state transition rate, to calculate the steady state unavailability rate for the system, and then the relay protection is quantitatively evaluated.

Two State Markov Model. The single unit repairable system has two states. $X(t)=0$ is the working state, $X(t)=1$ is the fault state. When the system starts to work, it will begin to transfer to the fault condition 1 with a certain probability. In per unit time, the probability transferred from state 0 to 1 is $\lambda$ and staying in state 0 with probability $1-\lambda$. When system achieves at fault state 1 , it may enter into the working state with constant repair rate ${ }^{\mu}$ due to repairing. In per unit time, the probability transferred from state 1 to state 0 is and staying in state 0 with probability $1-\mu$. Its all transferred status is as shown in the Figure 3-1 below:

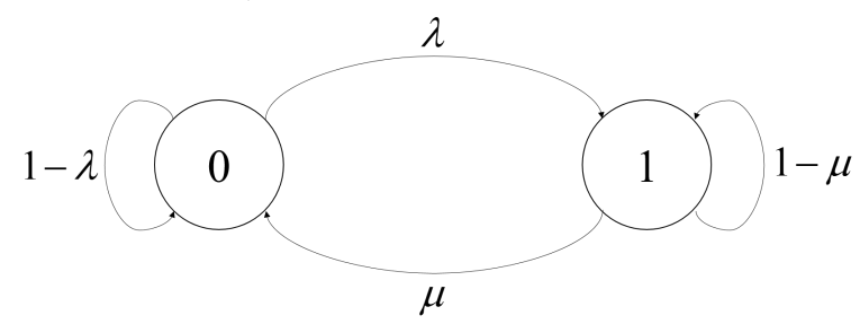

Figure 3-1 Two state Markov model

According to the state transition diagram, the transtion-probablity matrix $P$ is:

$$
P=\left[\begin{array}{cc}
1-\lambda & \lambda \\
\mu & 1-\mu
\end{array}\right](3-1)
$$

The transition density matrix $A$ is:

$$
A=P-I=\left[\begin{array}{cc}
-\lambda & \lambda \\
\mu & -\mu
\end{array}\right](3-2)
$$

The combined differential equations is:

$$
\left\{\begin{array}{l}
P_{0}^{\prime}(t)=-\lambda P_{0}(t)+\mu P_{1}(t) \\
P_{1}^{\prime}(t)=\lambda P_{0}(t)-\mu P_{1}(t)
\end{array}\right.
$$

Assuming that the system is in a normal state at the initial moment. There are initial conditions $P_{0}(0)=1, P_{1}(0)=0$.Giving the function (3-3) Laplace upper and the pullback transform: 


$$
\left\{\begin{array}{l}
A(t)=P_{0}(t)=\frac{\mu}{\lambda+\mu}+\frac{\lambda}{\lambda+\mu} e^{-(\lambda+\mu) t} \\
Q(t)=P_{1}(t)=\frac{\lambda}{\lambda+\mu}-\frac{\lambda}{\lambda+\mu} e^{-(\lambda+\mu) t}
\end{array}\right.
$$

$A(t)$ expresses the probabilitythat system is in the normal availability state at $t$ moment, called availability degree, And $Q(t)$ expresses the unavailability.

When the ${ }^{t}$ is $\rightarrow \infty$, the steady-state availability and unavailability[3] are obtained:

$$
\left\{\begin{array}{l}
A(\infty)=P_{0}(\infty)=\frac{\mu}{\lambda+\mu}(3-7) \\
Q(\infty)=P_{1}(\infty)=\frac{\lambda}{\lambda+\mu}
\end{array}\right.
$$

Four State Markov Model. Single unit repairable system simply considers a single component or a simple system abstracted into a single component. When the system is complex, this analysis is likely to cause relatively large deviation. Therefore, when the relay protection device repairing itself also considered, it can establish a four state Markov model to analyze the system reliability. The system model diagram is shown in Figure 3-2. Among them, the state 0 is the normal working state of the protection device. When the relay protection detects fault by itself, the state changes from 0 to I. After the restoration of the protection device, the state changes back to 0 . When the relay protection is not detected fault by itself, the state is transferred from 0 to II. At this time, only when device is operated by mistake or by regular maintenance can the protection fault be found. State III show the relay protection of the equipment a regular maintenance. When there are regular maintenance, the state changes from 0 to III. After completion of a regular overhaul, the state changes back to 0 .

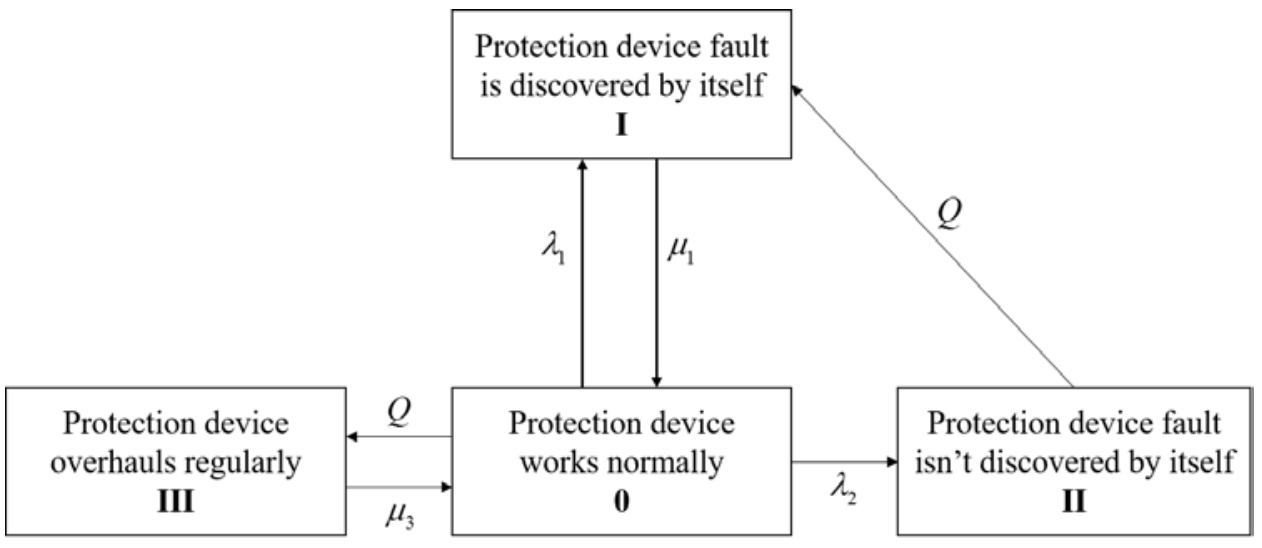

Figure 3-2 Four state Markov model

$\lambda_{1}$ The fault probability of self-repairing the relay protective device, sub/h;

$\lambda_{2}$ The fault probability without self-repairing the relay protective device, sub /h;

Q Regular maintenance rate, sub/h;

$\mu_{1}$ Repair rate after relay protection device failure by self-inspection founded, sub /h;

$\mu_{3}$ Repair rate of regular maintenance, sub /h;

If the system is exponential distribution or meets the description of the Markov model, the system availability rate with time changing: 


$$
A(t)=\frac{\mu}{\lambda+\mu}+\frac{\mu}{\lambda+\mu} e^{-(\lambda+\mu) t}
$$

If the system consists of $N$ parts in series, each part meets the Markov process. Assuming that the fault rate and repair rate of the $i$ state are $\lambda_{I}$ and $\mu_{I}$, so the system reliability degree is:

$$
R(t)=\exp \left(-\sum_{i=1}^{N} \lambda_{i} t\right)(3-11)
$$

The steady-state availability of the system is:

$$
A(\infty)=\frac{1}{1+\sum_{i=1}^{N} \lambda_{i} / \mu_{i}}
$$

\section{Solving the Optimal Maintenance Cycle Based on the Markov Model}

Based on the assumptions of constant failure rate ${ }^{[4]}$, the above mentioned two Markov model's unavailability rate can be calculated, the failure rate and the repair rate changing influence on the unavailability can be analyzed. And then it can calculate out the optimal preventive maintenance cycle of relay protection device and the optimal maintenance cycle under different fault rate of protection devices. Under the assumption of time varying fault efficiency, the Markov model is simulated by Matlab, and the effect of different maintenance strategies on the non-availability rate is analyzed.

In the study of the relay protection system reliability, the following assumptions are made:

The device fault can be detected when the maintenance is regularly scheduled or after the outage After the overhaul, the device can be restored to level in a good condition

In the process of maintenance, it won't lead to a new fault

The device must be stopped in the maintenance

The state space can be defined as the state transition matrix:

$$
A=\left[\begin{array}{cccc}
1-\left(\lambda_{1}+\lambda_{2}+Q\right) & \lambda_{1} & \lambda_{2} & Q \\
\mu_{1} & 1-\mu_{1} & 0 & 0 \\
0 & Q & 1-Q & 0 \\
\mu_{3} & 0 & 0 & 1-\mu_{3}
\end{array}\right](4-1)
$$

A The state transition matrix;

The resident probability matrix of 4 states is defined as

$$
P=\left[\begin{array}{llll}
P_{1} & P_{2} & P_{3} & P_{4}
\end{array}\right],\left(\sum_{i=1}^{4} P_{i}=1\right)(4-2)
$$

In this model, a new reliability index-the relay protection device non-availability is defined. It means all the relay protection working abnormally situations. That is the sum presence probability of four state Markov space model in the state I, II, III ${ }^{[5]}$. Based on the function (4-2) and (4-3), the relay protection device non-availability rate function is:

RelUn $=P_{2}+P_{3}+P_{4}(4-4)$

RelUn The device unavailable rate;

\section{Example Analysis}

The single and double protection device model parameters are selected based on a power grid actual data and the reference ${ }^{[6][7]}$. 
Through simulating the 3 state and 4 state model above the paper, we can obtain the operation time of different maintenance strategies for unavailable rate at different levels.

Because the equipment is more reliable at the beginning of operation, the change are not obvious, and the unavailable rate is low. We compare the cases of the unavailable rate firstly up to 0.01 after 2500 days. And the result is shown in Table 1.

Table 1 Operation time of 4-state model unavailability to reach 0.01

\begin{tabular}{cc}
\hline Maintenance strategy & Operation time/d \\
\hline maintenance partly once a year & 3480 \\
maintenance partly once every two years & 3376 \\
maintenance partly once every three years & 3303 \\
no maintenance & 2894 \\
maintenance partly once every six years & 3303 \\
maintenance partly once every nine years & 2474 \\
\hline
\end{tabular}

As the result shown in Table 1, the maintenance partly cycle is more frequent and the unavailable rate increased more slowly because of the equipment operating to the later stage in a fixed full inspection cycle for 6 years. Under the fixed part inspection period for 3 years, a full inspection cycle too long may lead to unavailable rate increase too fast for ahead of schedule to achieve the level of 0.01 .

\section{Conclusion}

Chinese rapid rise and development is inseparable from the electric energy. The safe operation of the power grid is particularly important. The task of the power system is as much as possible to supply the users for electronic economical and reliability. Relay protection plays an extremely important role in ensuring the safe system operation, preventing the occurrence of accidents and the expansion of the faults. With the continuous improvement of the power grid level, relay protection should avoid large area blackout as much as possible.

The main purpose of this paper is through establishing the mathematical model to analyze power system operation data and evaluate the reliability of relay protection device. And the paper obtains the complete algorithm of availability rate, graphical inspection and further optimization inspection at different repair rates.

\section{Reference}

[1] Xiang Gao, Application technology of relay protection condition based maintenance. Beijing, China Electric Power Press, 2008

[2]Bilintor R, Tatla J. Composite generation and transmission system adequency evaluation including protection system failure modes[J]. IEEE Trans on Power Apparatus and Systems, 1983, 102(6): 1823-1830.

[3] Maosheng Ding, Gang Wang, Wen he. Optimal maintenance interval time of relay protection based on reliability economic analysis [J]. Proceedings Of the Chinese Society Electrical Engineering, 2007, 27 (25): 44-47.

[4] Yongji Guo, Reliability Analysis Of Electric Power System [M]. Beijing, Tsinghua University Press, 2003

[5] Ke'e Zeng, Research on operation situation and reliability indices of protective relayings in power system[J]. Power System Technology, 2004, 28(14): 83-85(in chinese)

[6]WANG Ruichen, XUE Ancheng, BI Tianshu, et al. Timevarying failure rate estimation of relay 
protection devices and their regional differences analysis[J]. Automation of Electric Power Systems, 2012, 36(5): 11-15.

[7]XUE Ancheng, WANG Bao, WANG Ruichen, et al. Analysis of time-varying failure characteristics for HVDC protection devices[J]. Automation of Electric Power Systems, 2012,36(19); 17-20. 